\title{
Sleep Does Not Benefit Probabilistic Motor Sequence Learning
}

\author{
Sunbin Song, ${ }^{1,3}$ James H. Howard Jr, ${ }^{2,4}$ and Darlene V. Howard ${ }^{1,3}$ \\ Departments of ${ }^{1}$ Psychology and ${ }^{2}$ Neurology and ${ }^{3}$ Interdisciplinary Program in Neuroscience, Georgetown University, Washington, DC 20007, and \\ ${ }^{4}$ Department of Psychology, Catholic University, Washington, DC 20064
}

It has become widely accepted that sleep-dependent consolidation occurs for motor sequence learning based on studies using fingertapping tasks. Studies using another motor sequence learning task [the serial response time task (SRTT)] have portrayed a more nuanced picture of off-line consolidation, involving both sleep-dependent and daytime consolidation, as well as modifying influences of explicit awareness. The present study used a variant of the SRTT featuring probabilistic sequences to investigate off-line consolidation. Probabilistic sequences confer two advantages: first, spontaneous explicit awareness does not occur, and second, sequence learning measures are continuous, making it easier to separate general skill from sequence-specific learning. We found that sleep did not enhance general skill or sequence-specific learning. In contrast, daytime enhancement occurred for general skill but not for sequence-specific learning. Overall, these results suggest that motor learning does not always undergo consolidation with sleep.

Key words: sleep; daytime enhancement; memory consolidation; implicit learning; motor sequence learning; probabilistic sequences

\section{Introduction}

It is widely accepted that sleep-dependent consolidation occurs for motor learning (Stickgold, 2005). For example, motor sequence learning usually reveals sleep-related enhancement (Walker et al., 2002, 2003a). Most studies have used a fingertapping task in which subjects repeatedly tap out a known fixed sequence. Subjects are faster and more accurate after an off-line period but only if it includes sleep (Fischer et al., 2002; Walker et al., 2002, 2003a; Kuriyama et al., 2004; Manoach et al., 2004; Stickgold, 2005).

However, these finger-tapping tasks have a strong explicit component in that the subjects repeatedly type out a known sequence. Explicit sequence knowledge has been shown to modify off-line consolidation features (Robertson et al., 2004). In addition to their explicit nature, finger-tapping tasks are further limited because general motor skill and sequence-specific motor learning cannot be separated. For these reasons, studies using the serial response time task (SRTT) have been useful in revealing the nuances of off-line consolidation for motor sequence learning. In the SRTT, subjects press buttons in response to events, and sequence learning is shown by faster and more accurate performance on blocks in which the event sequence is fixed compared with random (Nissen and Bullemer, 1987). Learning on the SRTT can be implicit, in that subjects can show sequence learning without awareness. However, explicit sequence learning can be introduced with intentional instructions. Unfortunately, spontaneous explicit knowledge of the pattern can also occur with extended

Received May 5, 2007; revised Sept. 25, 2007; accepted Sept. 27, 2007.

This work was supported by National Institutes of Health Grants R37AG15450 and F31NS053388.

Correspondence should be addressed to Sunbin Song at the above address. E-mail: sss35@georgetown.edu.

DOI:10.1523/JNEUROSCI.2062-07.2007

Copyright $\odot 2007$ Society for Neuroscience $\quad$ 0270-6474/07/2712475-09\$15.00/0 practice even without intentional instruction (Robertson and Cohen, 2006).

For this reason, probabilistic sequences are often used instead of fixed (deterministic) ones. For probabilistic sequence learning paradigms, certain sequences occur at a higher frequency than others. This sequence structure confers two advantages: first, spontaneous explicit awareness is less likely to occur in incidental conditions, and second, sequence learning measures are continuous and not reliant on a comparison between patterned and random blocks. This makes it easier to separate general skill from sequence-specific learning.

In the following study, pattern awareness was also manipulated via cues and instruction that allowed for intentional learners to acquire explicit awareness of the pattern when cues were present (on cued blocks), but not when they were absent (on probe blocks) (Song et al., 2007). Our focus was on implicit learning, so blocks flanking off-line periods were always probe blocks for both incidental and intentional groups. However, the addition of an intentional group allowed us to also discern the modifying effects of explicit awareness on consolidation for implicit learning.

\section{Materials and Methods}

Subjects and design

All subjects signed informed consent.

Four experimental groups. Thirty-six right-handed volunteers (aged $18-25$ years; 16 males and 20 females) were recruited from Georgetown University, with each participant coming in for three visits. Two independent variables varied between subjects: time of first test and instruction. Eighteen subjects were tested in the morning first and 18 in the evening first, and all returned 12 and $24 \mathrm{~h}$ after initial testing. Those who had their first visit at 8:00 A.M. (day first) had $12 \mathrm{~h}$ of waking between their first and second visit, and $12 \mathrm{~h}$, which included sleep between their second and third visits. Those tested at 8:00 P.M. first (night first) had 
$12 \mathrm{~h}$, which included sleep between their first and second visits, and 12 waking hours between their second and third visits. In addition, onehalf of each group was given incidental instructions with no mention of the regularity. The other half was given intentional instructions, in which subjects were told that there was a $4 \mathrm{U}$ repeating alternating regularity, and that gray targets were pattern trials, and black targets were random trials. They were asked to explicitly learn the regularity followed by the gray targets.

Diurnal control groups. Eighteen additional right-handed volunteers (aged 18-25 years; 4 males and 16 females) were recruited to come in for two visits and serve in one of two diurnal control groups. All of these subjects were given incidental instructions. Nine were tested at 8:00 A.M. and retested $24 \mathrm{~h}$ later at 8:00 A.M., whereas the other nine were tested at 8:00 P.M. and retested $24 \mathrm{~h}$ later at 8:00 P.M. Thus, all diurnal control subjects had a waking day and a night of sleep in between test and retest, and all completed their retest at the same time-of-day as their original test.

\section{Testing procedure}

On each visit, subjects were given the alternating serial response time (ASRT) task (Howard and Howard, 1997). The version used here is more fully outlined by Song et al. (2007). In brief, participants responded by button press to a target (filled-in circle) that appeared in one of a row of four open circles. The circle remained filled in until participants pressed the correct key, at which time it was cleared and another circle filled in after a delay of $120 \mathrm{~ms}$. Each block began with 8 random trials (for warm-up), followed by 80 experimental trials. These 80 trials consisted of an eight-item sequence, in which pattern trials alternated with random trials (e.g., $1 \mathrm{r} 4 \mathrm{r} 3 \mathrm{r} 2 \mathrm{r}$ in which the numbers refer to positions 1, 2, 3, and 4, and "r" refers to a randomly chosen one of these four locations), and this eight-item sequence repeated 10 times in a block. There were five blocks in each epoch.

There were two types of epochs, cued or probe. On cued epochs, the pattern trials were gray targets and random trials were black targets. In the probe epochs, all trials were black targets, such that color did not differentiate between pattern and random trials. In the study by Song et al. (2007), it was shown that when subjects were given intentional instructions (told about the nature of the regularity for the gray targets in the cued epochs), they were able to acquire explicit knowledge of the repeating $4 \mathrm{U}$ pattern. In contrast, those given incidental instructions did not notice this pattern in the cued epochs. Neither those given intentional nor incidental instructions became aware of the pattern in the probe epochs although it was identical to the one found in the cued epochs. Thus, probe epochs measured implicit learning for all groups regardless of instruction, whereas cued epochs also reflected explicit sequence knowledge for intentional but not incidental groups.

On the first visit in the present study, subjects completed nine epochs of the ASRT. Figure 1 shows a schematic of the study design. Every third epoch was a probe epoch. On their second and (if applicable) third visits, subjects completed four epochs of the ASRT. For all visits, the first and last epochs were probe epochs. Thus, the experimental groups completed 17 epochs total, with probe epochs 9 and 10 flanking the first off-line period and probe epochs 13 and 14 flanking the second off-line period. Probe epochs were epochs $3,6,9,10,13,14$, and 17. Cued epochs were epochs $1,2,4,5,7,8,11,12,15$, and 16 . For a given subject, the pattern (e.g., 1r2r4r3r) remained the same through all epochs across visits. However, across subjects within each group, the pattern varied (five possible; $1 \mathrm{r} 2 \mathrm{r} 3 \mathrm{r} 4 \mathrm{r}$ was not used because of ease of discovery for intentional learners). $\begin{array}{lll}\text { Visit } 1 & \text { Visit } 2 & \text { Visit } 3\end{array}$
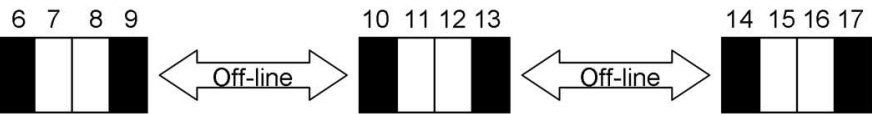

cidental Instruction):

$\begin{array}{lccccc}\text { Experimental } & \text { Groups } & \text { (Intentional or Incidental } & & & \\ \text { Night First: } & 8 \mathrm{pm} & \text { Sleep } & 8 \mathrm{am} & \text { Awake } & 8 \mathrm{pm} \\ \text { Day First: } & 8 \mathrm{am} & \text { Awake } & 8 \mathrm{pm} & \text { Sleep } & 8 \mathrm{am}\end{array}$

8pm
$8 \mathrm{am}$

roups (Incidental Instruction):

8am Wake + Sleep $\quad$ Sam

Sleep + Wake $8 p m$

Figure 1. Schematic of the study design. On the first visit, subjects completed nine epochs of the ASRT. Every third epoch,

Table 1. Characterization of sleep in groups

\begin{tabular}{llll}
$\begin{array}{l}\text { PSQI score } \\
\text { (0-21: } \text { no to severe } \\
\text { sleep disturbance) }\end{array}$ & $\begin{array}{l}\text { MEQ score } \\
\text { (16-86: A.M. to } \\
\text { P.M. preference) }\end{array}$ & $\begin{array}{l}\text { Hours of } \\
\text { intervening } \\
\text { sleep }\end{array}$ & $\begin{array}{l}\text { Quality of } \\
\text { intervening sleep } \\
\text { (1-10 self-assessed) }\end{array}$ \\
$6.1 \pm 1.8$ & $42.3 \pm 9.3$ & $5.4 \pm 1.6$ & $7.4 \pm 1.1$ \\
$5.7 \pm 2.2$ & $39.0 \pm 8.7$ & $6.1 \pm 1.8$ & $6.3 \pm 2.3$ \\
$5.8 \pm 1.8$ & $47.0 \pm 6.2$ & $5.4 \pm 2.0$ & $7.5 \pm 1.2$ \\
$5.5 \pm 2.3$ & $48.3 \pm 8.7$ & $5.9 \pm 0.8$ & $6.8 \pm 1.7$ \\
$5.8 \pm 2.3$ & $45.3 \pm 8.6$ & $6.7 \pm 1.3$ & $7.8 \pm 1.5$ \\
$5.7 \pm 1.9$ & $44.6 \pm 10.1$ & $8.0 \pm 1.4$ & $7.6 \pm 1.2$ \\
\hline
\end{tabular}

To assess explicit awareness, subjects were given the following tests: sequence report, generation, questionnaire, and card sorting [as outlined in more detail by Song et al. (2007)]. In brief: for sequence report, after each block, intentional subjects reported (or guessed) the pattern. On the final visit, at the end of the last session, both intentional and incidental subjects were given generation, questionnaire, and card sorting in that order. For the generation task, all subjects listed a typical sequence of 16 elements numerically, separately for cued and probe epochs. For card sorting, subjects sorted 64 cards, each with one triplet (three trials) sequence, into two piles, one for triplets they thought occurred more often and one for less often. Subjects were told to sort for probe epochs. Questionnaires tested for awareness by a series of questions containing increasingly specific questions about the sequence.

In addition, subjects filled out forms assessing their sleep quantity and quality if the intervening time period between visits was overnight. Because this study lacked polysomnographic recordings for sleep measures, all reports as to sleep duration and quality were subjective. For the daytime condition, napping was excluded by instruction and questionnaire, but otherwise, daytime activities were not regulated in any way. Subjects were also given a Morningness-Eveningness Questionnaire (MEQ) (Horne and Ostberg, 1976) and the Pittsburgh Sleep Quality Index (PSQI) sleep inventory (Buysse et al., 1989). A summary of this information can be found in Table 1. ANOVAs revealed no significant differences between the groups on these measures.

\section{Results}

In the ASRT, pattern trials alternate with random ones, causing certain triplets of trials to occur at a higher frequency than others. For example, for the pattern $1 \mathrm{r} 3 \mathrm{r} 2 \mathrm{r} 4 \mathrm{r}$, triplets beginning with 1 and ending with 3 are high frequency, whereas those beginning with 3 and ending with 1 are low frequency. In other words, 50\% of the trials can be predicted with complete certainty by the event on $n-2$ (pattern trials), and thus all pattern trials are high frequency. The other $50 \%$ of trials are random trials and there is no predictive value of the event on $n-2$. However, by chance, 

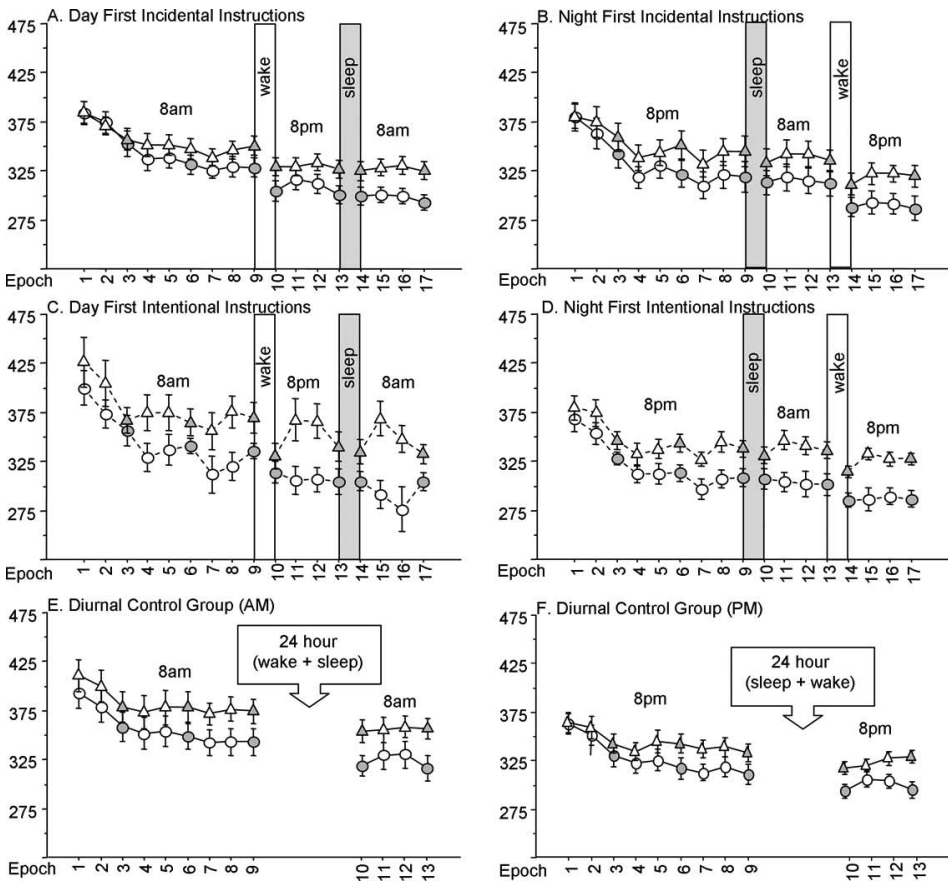

Figure 2. Median reaction times (for correct trials only) were determined separately for high (open triangles)- and low (open circles)-frequency triplet types for each epoch for each instruction group. Probe epochs have filled symbols and cued epochs have open symbols. For experimental groups, off-line periods are indicated by shaded or open boxes. The shaded boxes indicate a night of sleep. Epochs are plotted on the $x$-axis, and for intentional subjects, the lines are dotted, and for incidental subjects, the lines are solid. $\boldsymbol{A}$ and $\boldsymbol{B}$ plot day-first and night-first incidental subjects, and $\boldsymbol{C}$ and $\boldsymbol{D}$ plot day-first and night-first intentional subjects. $\boldsymbol{E}$ and $\boldsymbol{F}$ plot diurnal control groups. Error bars indicate SEM.

some of these may have the same triplet structure $(n-2, n-1$, $n)$ as pattern trials, and are thus also high frequency. Subjects become sensitive to relative triplet frequency as evidenced by the fact that performance improves on the third event if it falls within a high-frequency triplet versus a low-frequency triplet, regardless of whether this high-frequency triplet ends with a pattern or random trial (Howard et al., 2004). As opposed to deterministic sequences in which event $n-1$ is $100 \%$ predictive of event $n$, this sequence structure is probabilistic because certain triplet sequences occur at a higher frequency than others (five times more frequent). For results reported in the following sections, highand low-frequency triplet trials are compared, but we removed trills (i.e., 121) and repetitions (i.e., 111) from all analyses because they are always low frequency for all subjects, and so performance on them could reflect preexisting biases, rather than sequence-specific learning.

Sequence-specific learning was assessed by triplet type effects for reaction time (RT) and accuracy. The triplet type effect is a difference score contrasting high- and low-frequency triplets such that a larger difference signals more sequence-specific learning. In contrast, skill learning was assessed by overall reaction time and accuracy. This distinction is useful because we can separate overall performance gains from sequence-specific ones. Paradigms previously used in off-line enhancement studies cannot as easily separate these two different forms of learning.

The results are presented in the following order. First, explicit awareness tests are analyzed. These measures allow us to establish the implicit nature of learning in the incidental groups, and to characterize the awareness gained by the intentional subjects. Second, for experimental groups, motor responses from the first visit are analyzed to reveal how awareness, practice, and time-ofday influence learning measures. Third, for experimental groups,

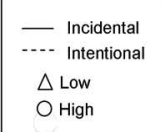

probe epochs flanking off-line periods (one over day, and one over night) are analyzed to characterize daytime- and sleeprelated enhancement for implicit learning. Fourth, for diurnal control groups, probe epochs flanking a $24 \mathrm{~h}$ off-line period are analyzed to assess off-line enhancement while controlling for time of day.

\section{Explicit awareness tests Incidental subjects}

No incidental subject showed awareness of the regularity as assessed by generation on cued epochs, generation on probe epochs, questionnaire, or card sorting for probe epochs (for the card sort, experimental groups: mean percentage correct, $50.4 ; \mathrm{SD}$, $7.2 ; t_{(16)}=0.2 ; p=0.84$; diurnal control groups: mean percentage correct, $49.0 ; \mathrm{SD}$, $\left.7.7 ; t_{(16)}=-0.5 ; p=0.61\right)$.

\section{Intentional subjects}

Intentional subjects were able to gain explicit awareness of the pattern on the cued epochs as revealed by their ability to report the complete pattern. These intentional subjects could describe this explicit knowledge on cued epochs as assessed by generation for cued epochs, and questionnaire. These same subjects reported no awareness of the regularity for probe epochs as assessed by questionnaire, or by generation for probe epochs, excepting four subjects (three from the awake-first and one from the sleep-first group) who were removed from the study because they revealed awareness on the questionnaire and generation. The remaining subjects in the intentional group ( $n=6$ awake first; $n=8$ sleep first) gained awareness of the pattern on the cued epochs on average on block $2 \pm 1$ of the first epoch, as assessed by sequence report. For these subjects, card sorting for high- and low-frequency triplet types for probe epochs was marginally above chance (mean percentage correct, $\left.54.1 ; \mathrm{SD}, 6.4 ; t_{(12)}=2.1 ; p<0.06\right)$. This suggests that, even when subjects cannot explicitly describe the pattern in the probe blocks, some very limited recognition may be achieved by the end of the extended training used here.

In general, these explicit awareness measures replicate the results by Song et al. (2007) and indicate that the probe epochs are tapping relatively pure sequence-specific implicit learning. This is especially clear for the incidental groups who gave no sign of any knowledge of the regularity on any of the explicit measures.

\section{Experimental groups}

Practice, awareness, time-of-day effects on motor learning (first visit)

To observe practice, awareness, and time-of-day effects, motor learning measures from the first visit were analyzed separately. Because only the first visit was included in these analyses, any effects observed cannot be attributed to off-line changes. Means of median reaction times for correct trials were determined separately for high- and low-frequency triplet types for each epoch for all four experimental groups. These values are represented by epochs 1 through 9 of Figure 2, $A-D$, which plots all 17 epochs. Figure 4 plots the difference between high- and low-frequency triplets (triplet type effects) for reac- 
tion time, which assesses sequencespecific learning. Accuracy was also determined for each triplet type for each instruction group. Mean accuracy for high- and low-frequency triplets across epochs is shown in Figure 3, $A-D$, and accuracy triplet type effects are shown in Figure 5.

\section{Reaction time}

The effects of instruction, time of day, and practice were examined in the following statistical analyses on reaction time measures. Reaction times for visit 1 were subjected to a mixed design instruction (incidental vs intentional) by epoch (1-9) by triplet type (high or low) by time of day (A.M. or P.M.) ANOVA. This revealed significant main effects of epoch $\left[F_{(8,224)}=\right.$ 98.1; mean square error (MSE), 263.6; $p<$ $0.0001]$, showing overall skill learning, and of triplet type $\left(F_{(1,28)}=72.1\right.$; MSE, 938.4; $p<0.0001)$. A triplet type by epoch interaction $\left(F_{(8,224)}=7.4\right.$; MSE, $132.4 ; p<$ $0.0001)$ indicated that sequence-specific learning occurred (Figs. 2, 4). A triplet type by instruction interaction $\left(F_{(1,28)}=8.5\right.$; MSE, 938.4; $p<0.007)$ indicated that, overall, intentional groups had a larger difference between high- and low-frequency triplets than did incidental groups. There was also a trend for a triplet type by instruction by epoch interaction $\left(F_{(8,224)}=\right.$ 1.9; MSE, 132.4; $p<0.06$ ) (Fig. 4).

To observe how explicit awareness affected learning, cued and probe epochs were analyzed separately. For the cued epochs, in addition to main effects of epoch $\left(F_{(5,140)}=121.3\right.$; MSE, 317.6; $\left.p<0.0001\right)$, triplet type $\left(F_{(1,28)}=36.1 ;\right.$ MSE, 1262.2; $p<0.0001)$, and a triplet type by epoch interaction $\left(F_{(5,140)}=12.6\right.$; MSE, 90.7; $p<$ $0.0001)$, there was a triplet type by instruction interaction $\left(F_{(1,28)}=7.4\right.$; MSE, $1262.2 ; p<0.02)$. As Figure 4 shows, this reflects the fact that the triplet type effect was greater for intentional than incidental subjects on the cued epochs, suggesting the intentional subjects were able to use their explicit sequence knowledge to speed responses to pattern trials (high-frequency trials are comprised of $\sim 80 \%$ pattern trials, and $20 \%$ random trials). Although Figure 4 suggests that the day first intentional subjects showed particularly high triplet type effects on cued blocks, in fact there were no significant time-of-day effects for cued epochs. This may be attributable to the large variability in the extent to which subjects used their explicit knowledge, as is apparent by the error bars for intentional subjects in Figure 4.

The analysis of the visit 1 probe epochs alone revealed significant main effects of epoch $\left(F_{(2,56)}=24.9\right.$; MSE, 144.0; $p<$ $0.0001)$, of triplet type $\left(F_{(1,28)}=201.1 ; \mathrm{MSE}, 110.2 ; p<0.0001\right)$, and a triplet type by epoch interaction $\left(F_{(2,56)}=12.2\right.$; MSE, 86.0; $p<0.0001)$, indicating that sequence-specific learning was occurring during the probe epochs on visit 1 . There was also a triplet
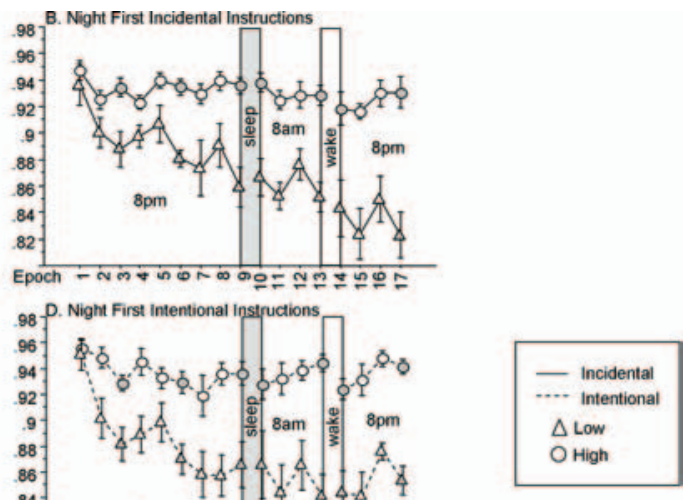

Figure 3. Mean accuracy scores were determined separately for high (open triangles) and low (open circles) triplet types for each epoch . Probe epochs have filled symbols and cued epochs have open symbols. Off-line periods are indicated by shaded night-first intentional subjects. $\boldsymbol{E}$ and $\boldsymbol{F}$ plot the two diurnal control groups. Error bars indicate $S E M$.
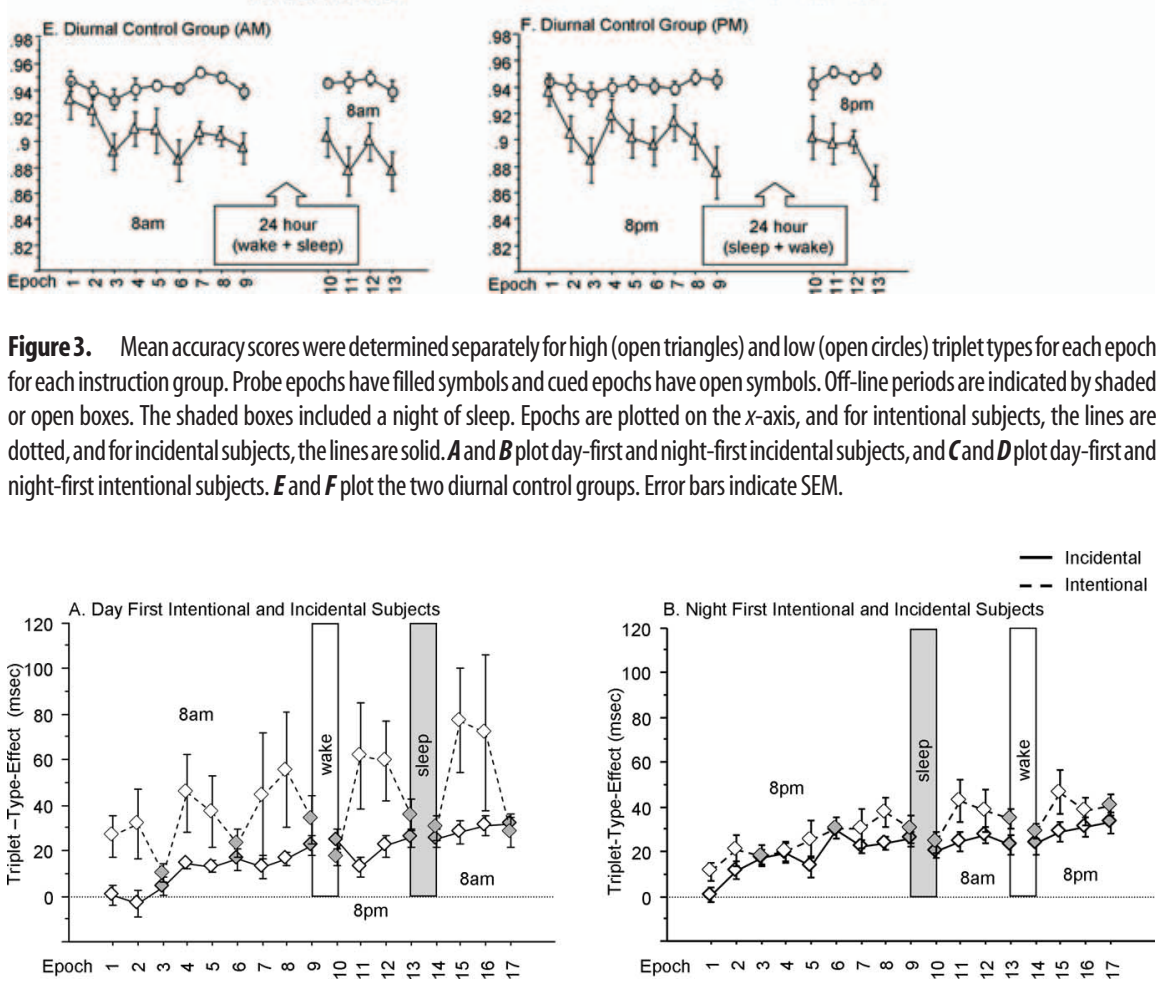

Figure 4. Sequence-specific learning for reaction time measures. The difference scores (triplet type effects; RT for low frequency minus RT for high frequency) for reaction time are plotted for the experimental groups. Probe epochs are plotted with filled plotted on the right. Incidental subjects are plotted with solid lines and intentional subjects are plotted with dotted lines. Triplet type effects increase with practice, but no off-line enhancement of sequence learning occurs. Error bars indicate SEM.

type by time-of-day interaction $\left(F_{(1,28)}=5.0\right.$; MSE, 110.2; $p<$ 0.04 ), indicating that on the first visit, subjects showed more implicit sequence-specific learning in the evening than the morning, although additional analysis showed that this effect was early in training and disappeared by the end of training (epoch $9 ; p=$ 0.88 ). There were no interactions or main effects of instruction on probe epochs, which is consistent with previous reports indicating that probe epochs are not influenced by explicit awareness (Song et al., 2007) (Fig. 3).

In summary, on visit 1 , reaction times indicated that implicit sequence-specific learning increased with practice, and that time of day did not have an effect on sequence learning or overall reaction time by the end of training on the first visit. Although intentional subjects showed more sequence learning on cued epochs than incidental subjects, sequence learning on probe epochs 

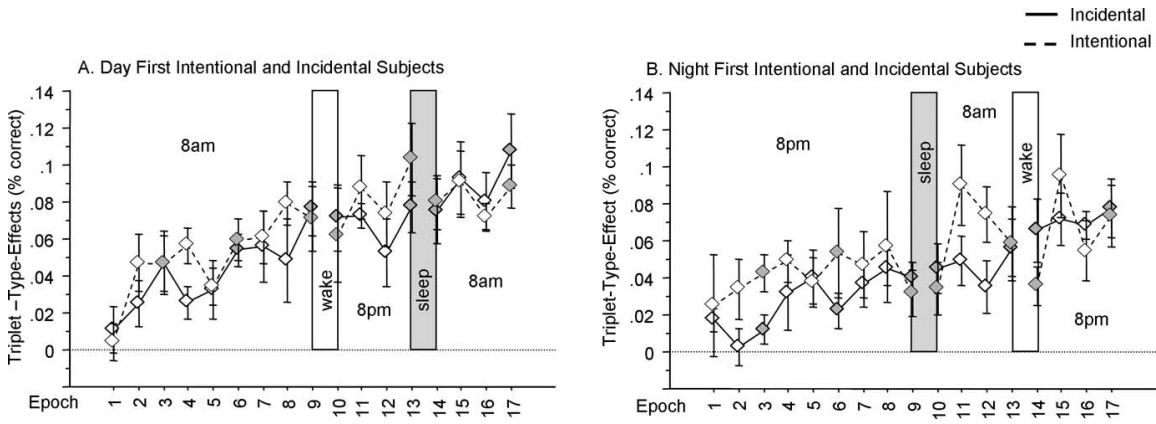

Figure 5. Sequence-specific learning for accuracy measures. The difference scores [triplet type effects; accuracy for high frequency minus accuracy for low frequency] for accuracy scores are plotted. Probe epochs are plotted with filled diamonds, and cued epochs are plotted with open diamonds. Day-first subjects are plotted on the left, and night-first subjects are plotted on the right. Incidental subjects are plotted with solid lines, and intentional subjects are plotted with dotted lines. Triplet type effects increase with practice, but no off-line enhancement of sequence learning occurs. Error bars indicate SEM.
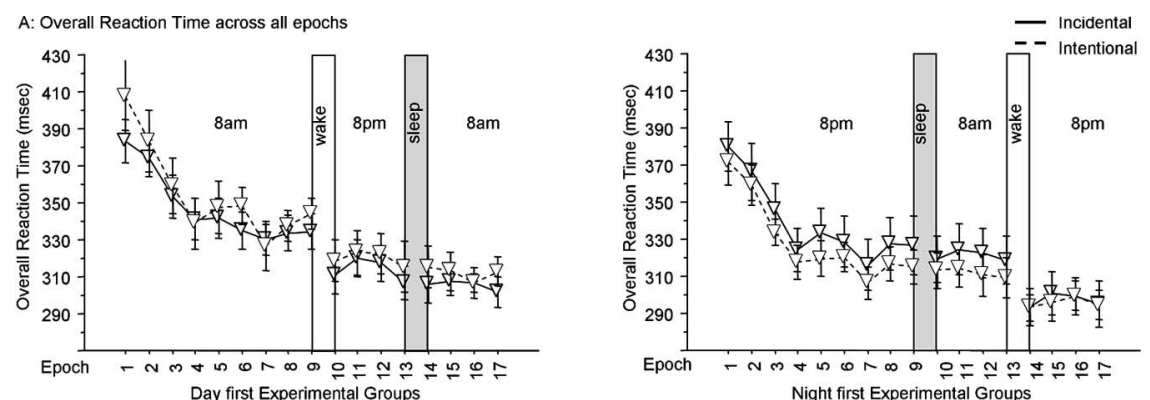

B: Changes in overall reaction time across offline periods
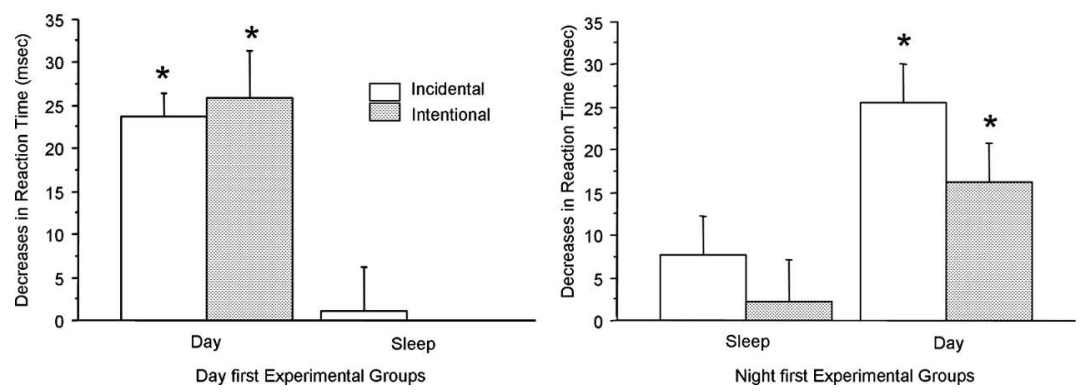

Figure 6. Daytime enhancement occurs for overall reaction time. $\boldsymbol{A}$, Probe epochs are plotted with filled triangles, and cued epochs are plotted with open triangles. Incidental subjects are plotted with solid lines, and intentional subjects are plotted with dotted lines. Day-first subjects are plotted on the left, and night-first subjects are on the right. $\boldsymbol{B}$, Overall reaction time change scores are calculated for each off-line period for each experimental group. Significant changes in overall reaction time only occur over the day. Error bars indicate SEM. Asterisks denote a significant difference of $p<0.01$.

was identical for incidental and intentional subjects, a finding that replicates our previous results (Song et al., 2007) and demonstrates that triplet type effects on probe epochs measure implicit sequence-specific learning for these incidental and intentional subjects.

\section{Accuracy}

The same analyses were conducted on accuracy. An ANOVA of first day accuracy (shown in Fig. 3) revealed significant main effects of epoch $\left(F_{(8,224)}=6.7\right.$; MSE, $\left.0.001 ; p<0.0001\right)$ indicating that accuracy fell across epochs (which as seen in Fig. 3, reflects falling accuracy for low-frequency triplets), of triplet type $\left(F_{(1,28)}=89.8\right.$; MSE, $\left.0.003 ; p<0.0001\right)$, and a triplet type by epoch interaction $\left(F_{(8,224)}=4.7\right.$; MSE, 0.001; $\left.p<0.0001\right)$ (sequence-specific learning) (Fig. 5). In contrast to reaction time, accuracy showed no effect of instruction, which is consistent with previous reports (Song et al., 2007). There were no time-of-day effects for accuracy.

Off-line changes over day and night

To observe off-line changes, reaction time and accuracy for probe epochs flanking both $12 \mathrm{~h}$ off-line periods were compared (epoch 9 vs 10; epoch 13 vs 14). We looked for off-line changes for both sequencenonspecific general skill learning, as reflected in overall reaction time and accuracy, and also for sequence-specific learning as reflected in triplet type effects.

\section{Off-line general skill learning}

Means of median reaction times for correct trials only are plotted in Figure 6A (and in Fig. 2 split by triplet type). Off-line changes in overall reaction time were computed by comparing the epoch right before and right after each off-line period (probe epochs). These off-line overall RT change scores for both off-line periods are plotted for the four experimental groups in Figure 6B. A mixed design instruction (incidental vs intentional) by sleep status (day first or night first) by off-line period ( 1 or 2) ANOVA on these overall reaction time change scores revealed only a significant sleep status by off-line period interaction $\left(F_{(1,56)}=31.7\right.$; MSE, 198.2; $p<0.0001)$. There were no significant effects of instruction indicating that instruction did not influence off-line changes on these probe epochs. Subsequent $t$ tests confirmed that only off-line periods over the daytime led to significant speeding in overall reaction time for each group (Fig. $6 \mathrm{~B}$, Table 2 ). In contrast, no off-line changes were found for overall accuracy (Fig. 3).

\section{Off-line sequence-specific learning}

For reaction time, off-line changes were calculated by comparing triplet type effects for the probe epochs flanking each off-line period. A mixed design instruction (incidental vs intentional) by sleep status (day first or night first) by off-line period (1 or 2) ANOVA on triplet type effect change scores for reaction time revealed no significant effects. One-sample $t$ tests confirmed that no off-line changes in triplet type effects for reaction time occurred for any condition (Table 2). Thus, there was no off-line enhancement for sequence-specific learning regardless of awareness or day/night differences. Off-line changes in triplet type effects for accuracy were computed by comparing the epochs flanking each off-line period as with reaction time. No off-line enhancement was found for sequence-specific accuracy measures.

In summary, these analyses revealed that general skill learning underwent daytime but not sleep enhancement. In contrast, sequencespecific implicit learning showed no off-line enhancement at all.

\section{Diurnal control groups}

Two diurnal control groups were added to rule out the possibility that time of day was contributing to or masking off-line enhance- 
Table 2. Daytime enhancement only occurs for overall reaction time

\begin{tabular}{lcccc}
\hline Instruction & Daytime change & Overnight change & Daytime change & \multicolumn{1}{c}{$\begin{array}{l}\text { Overnight change } \\
\text { sequence-specific (ms) }\end{array}$} \\
\hline sequence-specific (ms)
\end{tabular}

Asterisks denote a significant difference of $p<0.01$.

ments. By testing and retesting at the same time of day, any effects could be attributed to off-line processes and not to time of day. However, as each $24 \mathrm{~h}$ period consisted of both a wake and sleep period, it was not possible to attribute enhancements to sleep or day. Reaction time values for both diurnal control groups are represented in Figure 2, $E$ and $F$, and accuracy is shown in Figure $3, E$ and $F$. To observe off-line changes, reaction time and accuracy for probe epochs flanking the $24 \mathrm{~h}$ off-line period were compared.

\section{Off-line general skill learning}

Off-line changes in overall reaction time were computed by comparing the epoch right before and right after the off-line period (probe epochs 9 vs 10). $t$ tests confirmed that $24 \mathrm{~h}$ off-line periods led to significant speeding in overall reaction time for both the P.M. and the A.M. control group (Table 2, Fig. 7). In contrast, no off-line changes were found for overall accuracy.

\section{Off-line sequence-specific learning}

For reaction time, off-line changes were calculated by comparing triplet type effects for the probe epochs flanking each off-line period. $t$ tests confirmed that no off-line changes in triplet type effects for reaction time occurred for either P.M. or A.M. control groups (Table 2, Fig. 7). No off-line enhancement was found for sequence-specific accuracy measures.

In summary, diurnal control groups revealed that when time-of-day is kept constant, off-line enhancement in overall reaction time still occurs, whereas off-line enhancement for sequence-specific measures does not. A summary of the results across experimental and diurnal control groups is presented in Figure 7.

\section{Discussion}

\section{General summary}

Although it has become accepted that sleep-dependent consolidation occurs for motor sequence learning (Stickgold, 2005), our data suggest this is not always the case. Unlike past studies, we used a probabilistic sequence learning paradigm in which relatively pure implicit learning could be isolated on probe epochs, whereas explicit awareness could be limited to cued epochs in intentional learners. In addition, learning measures were continuous and sequence-specific learning could be separated from general skill learning.

Two main findings emerged. First, daytime enhancement occurred for overall reaction time, but not for sequencespecific learning. Second, in contrast to previous findings,
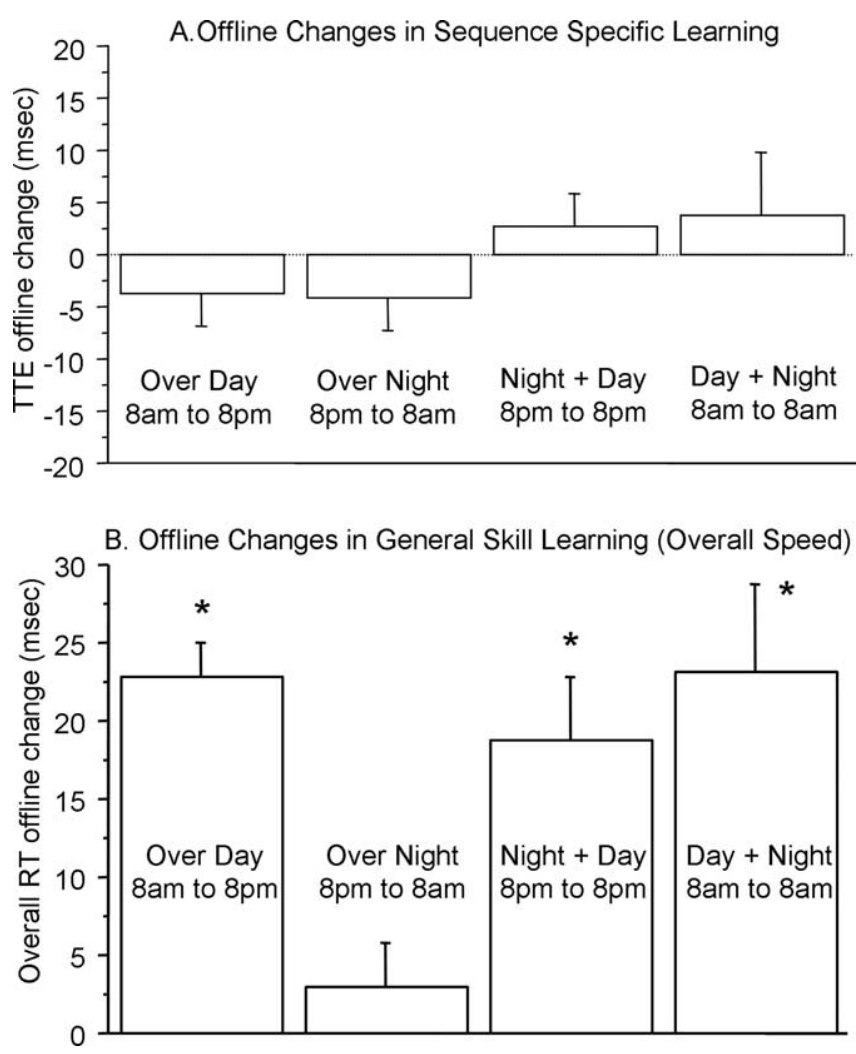

Figure 7. Summary of findings. Because findings were similar for all four experimental groups (total $n=32$ ), the data from all experimental groups were combined to generate averages for sequence-specific $(\boldsymbol{A})$ and general skill enhancements $(\boldsymbol{B})$ for off-line periods over the day or over a night of sleep. These were plotted next to results from the diurnal control groups. Statistically significant off-line changes are indicated by asterisks. Experimental groups only show enhancements for overall reaction time and only over the day, whereas diurnal groups also show enhancements for overall reaction time, but not for sequence-specific learning, over the $24 \mathrm{~h}$ off-line period. Error bars indicate SEM.

sleep-dependent consolidation did not occur for either general skill or sequence-specific learning.

\section{Daytime-related enhancement}

Consistent with SRTT studies (Robertson et al., 2004, 2005; Cohen et al., 2005; Press et al., 2005; Cohen and Robertson, 2007), we found a dissociation between daytime- and sleep-related pro- 
cesses. Enhancement in overall speed occurred only if the off-line period was over the day and not when it was overnight. These daytime improvements could have emerged from the dissipation of fatigue; however, this would suggest that daytime helps dissipate fatigue and a night of sleep does not, which is unlikely. These off-line changes in overall speed were not attributable to time-ofday effects: diurnal control groups who were tested and retested at the same time of day showed the same decreases in speed, regardless of whether they were tested and retested in the morning or evening.

Daytime consolidation has been shown to depend on the primary motor cortex (Robertson et al., 2005) and linked to specific motor movements (Cohen et al., 2005). Our findings are consistent with this conclusion in that contributions from motor domains would lead to general speeding. However, unlike findings from previous serial response time studies in which enhancements are often seen for both overall speed and sequence-specific learning, the consolidation here was only for general skill and not for sequence-specific learning. There are several possible explanations for our different findings.

First, previously used SRTT paradigms were not well suited to separate general speed decreases from sequence-specific ones. Second, spontaneous explicit awareness is more likely to occur for deterministic sequences (Schvaneveldt and Gomez, 1998; Jimenez and Mendez, 1999; Howard et al., 2004; Jimenez et al., 2006). Therefore, learning measures for deterministic sequences may reflect the use of explicit knowledge. Lastly, general skill and sequence-specific learning may both display daytime enhancements, but the latter only for deterministic sequences.

Daytime enhancement in overall speed does not occur for finger-tapping tasks. Finger-tapping tasks lack a perceptualmotor component in that motor responses depend solely on explicit representation to motor outputs, because subjects push keys in a remembered sequence without any external visual stimulus. However, a type of daytime consolidation does occur in the form of decreased susceptibility to interference by another sequence (Walker et al., 2003b), a finding that led researchers to conclude that daytime was involved in stabilization whereas sleep was related to enhancement (Walker, 2005). Our results show the opposite pattern, with daytime associated with enhancement of general skill, and sleep associated with no such enhancement. Subtle differences between tasks may be accounting for varying manifestations of off-line processes.

\section{Lack of sleep-related effects}

One study using probabilistic sequences (Fischer et al., 2007) found overnight improvement in adults, but overnight deterioration in children. Unlike this work, in which practice and circadian factors confounded off-line change measures, the present study included continuous learning measures and diurnal control groups to show a clear lack of sleep-dependent enhancement for probabilistic sequences. This finding is in contrast to studies using deterministic and/or explicit sequences (Walker et al., 2002, 2003a; Robertson et al., 2004, 2005; Cohen et al., 2005; Stickgold, 2005). There are two likely explanations for our different findings (i.e., the lack of explicit awareness in our study and/or the level of hippocampal involvement in probabilistic vs deterministic sequences).

Regarding awareness, when sequence-specific knowledge is explicit, as it is in finger-tapping tasks and in the SRTT for intentional learners, subjects repeat a known sequence. It is possible that sleep-related enhancement only occurs for such explicit motor sequence learning. Subjects labeled "implicit" learners in previous SRTT studies might have shown sleeprelated enhancement, because a limited amount of explicit awareness occurs spontaneously for deterministic sequences. Although those with significant explicit awareness are usually excluded, a limited amount of explicit awareness is still allowed for incidental subjects (Robertson et al., 2004). Interestingly, in a study conducted using the SRTT, sleep led to more explicit awareness of an implicitly acquired sequence, but did not enhance implicit motor sequence learning (Fischer et al., 2006). One other study has shown that sleep promotes explicit insight of a hidden abstract rule underlying a cognitive task (Wagner et al., 2004). Explicit awareness has been shown to significantly decrease reaction time for pattern trials (Willingham and Goedert-Eschmann, 1999), so an increase in explicit sequence knowledge would contribute to decreased reaction time on SRTT tasks. This argument might seem to conflict with the fact that, in our study, even in those with explicit awareness of the pattern (intentional), we found no sleep-dependent enhancement. However, in our intentional groups, we assessed only implicit learning, in that we compared probe epochs flanking the off-line periods (times when explicit awareness was not present in either group). Cued epochs did not flank off-line periods, and in addition, the extent to which subjects used their explicit knowledge varied, making it difficult to access off-line consolidation for explicit sequence learning. However, our data do suggest that when awareness cannot be used to affect reaction times (i.e., on probe epochs), awareness has no effect on sleep-dependent consolidation.

The second possible explanation for the lack of sleepdependent enhancement in the present study is that because deterministic sequences are more likely to involve the hippocampus than probabilistic sequences, the differential level of hippocampal involvement may determine whether sleep results in performance enhancement. A recent study gives direct evidence that slow oscillations during early slow-wave sleep enhance hippocampus-dependent learning (Marshall et al., 2006), and a widely held theory states that hippocampal replay underlies the sleep-dependent enhancements seen across many different tasks (Pavlides and Winson, 1989; Wilson and McNaughton, 1994; Peigneux et al., 2004; Ji and Wilson, 2007; Rasch et al., 2007). Probabilistic learning has been shown to recruit the striatum, and disengage the hippocampus (Poldrack et al., 2001). In contrast, there is some evidence that implicit learning for deterministic sequences involves the hippocampus (Curran, 1997; Schendan et al., 2003). This hippocampal-based argument is consistent with a study by Spencer et al. (2006), who found sleep consolidation for implicit learning only when there was a contextual component (two correlated cues) that added a hippocampal component. Thus, a highly likely explanation is that the degree of hippocampal involvement accounts for the differing sleep effects; deterministic sequence learning involves the hippocampus and is enhanced with sleep, but probabilistic sequence learning deactivates the hippocampus and hence does not show sleep-dependent enhancement.

The present study replicated previous findings that reaction time and not accuracy reflected the use of explicit knowledge (Song et al., 2007). Reaction time/accuracy dissociations have been seen in the sleep consolidation literature, because accuracy and reaction time have different sleep-related con- 
solidation properties (Stickgold, 2005). Regardless, in the current study, neither accuracy nor reaction time measures showed any sleep-related enhancement.

Although a widely held generalized theory states that procedural processes are consolidated with sleep, our study suggests that this may not always be the case for motor learning. For perceptual processes, a strong body of evidence suggests that sleep-dependent enhancement occurs (Karni et al., 1994; Gais et al., 2000; Stickgold et al., 2000a,b,c; Mednick et al., 2002, 2003; Atienza et al., 2004; Stickgold, 2005; Walker et al., 2005). However, for motor learning, the case for sleepdependent consolidation is weaker. Sleep-dependent enhancement is not consistently found for implicit motor learning in studies using the standard SRTT (Goedert and Willingham, 2002; Fischer et al., 2006; Spencer et al., 2006) nor for mirror tracing (Tucker et al., 2006). Motor adaptation has shown consistent sleep-dependent consolidation (Maquet et al., 2003; Huber et al., 2004; Backhaus and Junghanns, 2006), but in motor adaptation tasks, subjects learn to adjust motor movements in response to visual feedback. Thus, there is a large perceptual component. Overall, our results suggest that it is worth revisiting the issue of sleep-dependent consolidation for motor skills. In doing so, it will be important to distinguish between implicit and explicit motor learning, and to isolate general skill learning as reflected in overall response time, from sequence-specific learning. Lastly, it will be important to consider and parse out the various cognitive components and the brain systems involved in the acquisition and consolidation of a procedural skill. Because skill learning is often an amalgam of different processes, deciphering the offline consolidation features of each component will make it possible to better understand and use off-line performance enhancements (Robertson and Cohen, 2006).

\section{References}

Atienza M, Cantero JL, Stickgold R (2004) Posttraining sleep enhances automaticity in perceptual discrimination. J Cogn Neurosci 16:53-64.

Backhaus J, Junghanns K (2006) Daytime naps improve procedural motor memory. Sleep Med 7:508-512.

Buysse DJ, Reynolds III CF, Monk TH, Berman SR, Kupfer DJ (1989) The Pittsburgh Sleep Quality Index: a new instrument for psychiatric practice and research. Psychiatry Res 28:193-213.

Cohen DA, Robertson EM (2007) Motor sequence consolidation: constrained by critical time windows or competing components. Exp Brain Res 177:440-446.

Cohen DA, Pascual-Leone A, Press DZ, Robertson EM (2005) Off-line learning of motor skill memory: a double dissociation of goal and movement. Proc Natl Acad Sci USA 102:18237-18241.

Curran T (1997) Higher-order associative learning in amnesia: evidence from the serial reaction time task. J Cogn Neurosci 9:522-533.

Fischer S, Hallschmid M, Elsner AL, Born J (2002) Sleep forms memory for finger skills. Proc Natl Acad Sci USA 99:11987-11991.

Fischer S, Drosopoulos S, Tsen J, Born J (2006) Implicit learning-explicit knowing: a role for sleep in memory system interaction. J Cogn Neurosci 18:311-319.

Fischer S, Wilhelm I, Born J (2007) Developmental differences in sleep's role for implicit off-line learning: comparing children with adults. J Cogn Neurosci 19:214-227.

Gais S, Plihal W, Wagner U, Born J (2000) Early sleep triggers memory for early visual discrimination skills. Nat Neurosci 3:1335-1339.

Goedert KM, Willingham DB (2002) Patterns of interference in sequence learning and prism adaptation inconsistent with the consolidation hypothesis. Learn Mem 9:279-292.

Horne JA, Ostberg O (1976) A self-assessment questionnaire to determine morningness-eveningness in human circadian rhythms. Int J Chronobiol 4:97-110.

Howard DV, Howard Jr JH, Japikse K, DiYanni C, Thompson A, Somberg R
(2004) Implicit sequence learning: effects of level of structure, adult age, and extended practice. Psychol Aging 19:79-92.

Howard Jr JH, Howard DV (1997) Age differences in implicit learning of higher order dependencies in serial patterns. Psychol Aging 12:634-656.

Huber R, Ghilardi MF, Massimini M, Tononi G (2004) Local sleep and learning. Nature 430:78-81.

Ji D, Wilson MA (2007) Coordinated memory replay in the visual cortex and hippocampus during sleep. Nat Neurosci 10:13-15.

Jimenez L, Mendez C (1999) Which attention is needed for implicit sequence learning? J Exp Psychol Learn Mem Cogn 25:236-259.

Jimenez L, Vaquero JM, Lupianez J (2006) Qualitative differences between implicit and explicit sequence learning. J Exp Psychol Learn Mem Cogn 32:475-490.

Karni A, Tanne D, Rubenstein BS, Askenasy JJ, Sagi D (1994) Dependence on REM sleep of overnight improvement of a perceptual skill. Science 265:679-682.

Kuriyama K, Stickgold R, Walker MP (2004) Sleep-dependent learning and motor-skill complexity. Learn Mem 11:705-713.

Manoach DS, Cain MS, Vangel MG, Khurana A, Goff DC, Stickgold R (2004) A failure of sleep-dependent procedural learning in chronic, medicated schizophrenia. Biol Psychiatry 56:951-956.

Maquet P, Schwartz S, Passingham R, Frith C (2003) Sleep-related consolidation of a visuomotor skill: brain mechanisms as assessed by functional magnetic resonance imaging. J Neurosci 23:1432-1440.

Marshall L, Helgadottir H, Molle M, Born J (2006) Boosting slow oscillations during sleep potentiates memory. Nature 444:610-613.

Mednick S, Nakayama K, Stickgold R (2003) Sleep-dependent learning: a nap is as good as a night. Nat Neurosci 6:697-698.

Mednick SC, Nakayama K, Cantero JL, Atienza M, Levin AA, Pathak N, Stickgold R (2002) The restorative effect of naps on perceptual deterioration. Nat Neurosci 5:677-681.

Nissen MJ, Bullemer P (1987) Attentional requirements of learning: evidence from performance measures. Cogn Psychol 19:1-32.

Pavlides C, Winson J (1989) Influences of hippocampal place cell firing in the awake state on the activity of these cells during subsequent sleep episodes. J Neurosci 9:2907-2918.

Peigneux P, Laureys S, Fuchs S, Collette F, Perrin F, Reggers J, Phillips C, Degueldre C, Del Fiore G, Aerts J, Luxen A, Maquet P (2004) Are spatial memories strengthened in the human hippocampus during slow wave sleep? Neuron 44:535-545.

Poldrack RA, Clark J, Pare-Blagoev EJ, Shohamy D, Creso Moyano J, Myers C, Gluck MA (2001) Interactive memory systems in the human brain. Nature 414:546-550.

Press DZ, Casement MD, Pascual-Leone A, Robertson EM (2005) The time course of off-line motor sequence learning. Brain Res Cogn Brain Res 25:375-378.

Rasch B, Buchel C, Gais S, Born J (2007) Odor cues during slow-wave sleep prompt declarative memory consolidation. Science 315:1426-1429.

Robertson EM, Cohen DA (2006) Understanding consolidation through the architecture of memories. Neuroscientist 12:261-271.

Robertson EM, Pascual-Leone A, Press DZ (2004) Awareness modifies the skill-learning benefits of sleep. Curr Biol 14:208-212.

Robertson EM, Press DZ, Pascual-Leone A (2005) Off-line learning and the primary motor cortex. J Neurosci 25:6372-6378.

Schendan HE, Searl MM, Melrose RJ, Stern CE (2003) An FMRI study of the role of the medial temporal lobe in implicit and explicit sequence learning. Neuron 37:1013-1025.

Schvaneveldt RW, Gomez RL (1998) Attention and probabilistic sequence learning. Psychol Res 61:175-190.

Song S, Howard Jr JH, Howard DV (2007) Implicit probabilistic sequence learning is independent of explicit awareness. Learn Mem 14:167-176.

Spencer RM, Sunm M, Ivry RB (2006) Sleep-dependent consolidation of contextual learning. Curr Biol 16:1001-1005.

Stickgold R (2005) Sleep-dependent memory consolidation. Nature 437:1272-1278

Stickgold R, Whidbee D, Schirmer B, Patel V, Hobson JA (2000a) Visual discrimination task improvement: a multi-step process occurring during sleep. J Cogn Neurosci 12:246-254.

Stickgold R, Malia A, Maguire D, Roddenberry D, O'Connor M (2000b) Replaying the game: hypnagogic images in normals and amnesics. Science 290:350-353. 
Stickgold R, James L, Hobson JA (2000c) Visual discrimination learning requires sleep after training. Nat Neurosci 3:1237-1238.

Tucker MA, Hirota Y, Wamsley EJ, Lau H, Chaklader A, Fishbein W (2006) A daytime nap containing solely non-REM sleep enhances declarative but not procedural memory. Neurobiol Learn Mem 86:241-247.

Wagner U, Gais S, Haider H, Verleger R, Born J (2004) Sleep inspires insight. Nature 427:352-355.

Walker MP (2005) A refined model of sleep and the time course of memory formation. Behav Brain Sci 28:51-64.

Walker MP, Brakefield T, Morgan A, Hobson JA, Stickgold R (2002) Practice with sleep makes perfect: sleep-dependent motor skill learning. Neuron 35:205-211.

Walker MP, Brakefield T, Seidman J, Morgan A, Hobson JA, Stickgold R (2003a) Sleep and the time course of motor skill learning. Learn Mem 10:275-284.

Walker MP, Brakefield T, Hobson JA, Stickgold R (2003b) Dissociable stages of human memory consolidation and reconsolidation. Nature 425:616-620.

Walker MP, Stickgold R, Jolesz FA, Yoo SS (2005) The functional anatomy of sleep-dependent visual skill learning. Cereb Cortex $15: 1666-1675$

Willingham DB, Goedert-Eschmann K (1999) The relation between implicit and explicit learning: evidence for parallel development. Psychol Sci 10:531-534.

Wilson MA, McNaughton BL (1994) Reactivation of hippocampal ensemble memories during sleep. Science 265:676-679. 